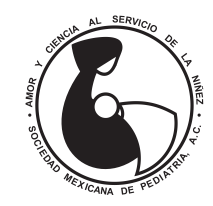

\title{
Niveles normales del marcador lipocalina asociada con gelatinasa de neutrófilo (NGAL) en orina en recién nacidos pretérmino y de término a las 24 y 72 horas de vida
}

\author{
Karen Plascencia-Pimentel, ${ }^{1,}{ }^{*}$ Isabel Bernárdez-Zapata, ${ }^{2}$ Moisés Noé Gerardo-Del Hoyo, ${ }^{2}$ \\ José Iglesias-Leboreiro, ${ }^{2}$ Mario Enrique Rendón-Macías, ${ }^{3}$ José Carlos Romo-Vázquez, ${ }^{4}$ \\ Juan Vicencio-Rivas, ${ }^{3}$ Corinne Michelle Lozano-Duau ${ }^{1}$ \\ ${ }^{1}$ Facultad Mexicana de Medicina, Universidad La Salle; ${ }^{2}$ Departamento de Pediatría y Neonatología Hospital Español de \\ México; ${ }^{3}$ Unidad de Investigación en Análisis y Síntesis de la Evidencia, Instituto Mexicano del Seguro Social; \\ ${ }^{4}$ Departamento de Nefrología del Hospital Infantil de México Federico Gómez, Secretaría de Salud, Ciudad de México.
}

\begin{abstract}
RESUMEN
Introducción: El biomarcador lipocalina asociada a gelatinasa de neutrófilo (NGAL) en orina presenta alta excreción en caso de isquemia de células renales del asa de Henle y túbulo colector; hasta ahora los valores normales en neonatos son controversiales. Objetivos: Presentar los valores de NGAL urinarios en neonatos sanos a las 24 y 72 horas de vida, según grupos de semanas de edad gestacional (SEG). Material y métodos: 70 neonatos (25-32 SEG, $n$ $=25$; 33-35 SEG, $n=25$; 36-38 SEG, $n=10$; 39-41 SEG, $\mathrm{n}=10)$. Los niveles de NGAL ( $\mathrm{ng} / \mathrm{mL}$ ) urinarios se determinaron por quimioluminiscencia. Los valores se muestran como mediana y valores mínimo y máximo (mediana [min - max]). Resultados: Los niveles de NGAL fueron diferentes según grupos de edad gestacional, tanto a las 24 horas como a las 72 horas. A las 24 horas $=25-32$ SEG $=18.2$ [0.5-543.1], 33-35 SEG = 22.3 [0.9-84.4], 36-38 SEG = 10.1 [2.5-181.9], 39-41 SEG = 20.4 [0-577]; a las 72 horas $=25-32$ SEG $=47.2[7.5-441.2], 33-35 \mathrm{SEG}=33.0$ [4.7-235.5], 36-38 $\mathrm{SEG}=27.7$ [4.09-173.1] 39-41 SEG = 17.8 [2.0-113.5] Conclusión: Los valores de NGAL urinarios normales en
\end{abstract}

\begin{abstract}
Introduction: The biomarker urinary neutrophil gelatinaseassociated lipocalin (U-NGAL) has high excretion in case of ischemia the renal cell loop of Henle and collecting tubule; so far the normal values in neonates are controversial. Objectives: To determine u-NGAL values in healthy newborns at 24 and 72 hours of life, according to weeks of gestation (WG). Material and methods: Seventy neonates (25-32 WG $n=25,33-35 W G n=25$ y 36-38 WG $n=$ $10 ; 39-41 \mathrm{WG}, \mathrm{n}=10)$. The $u-N G A L(\mathrm{ng} / \mathrm{mL})$ levels were determined by chemiluminescence. Values are shown as median as well as minimum and maximum (median [min max]). Results: u-NGAL levels were different in gestational age group at 24 and 72 hours. 24 hours $=25-32 W G=18.2$ [0.5-543.1], 33-35 WG = 22.3 [0.9-84.4], 36-38 WG $=10.1$ $[2.5-181.9], 39-41 \mathrm{WG}=20.4[0-577] ; 72$ hours $=25-32$ $W G=47.2$ [7.5-441.2], 33-35 WG = 33.0 [4.7-235.5], 36$38 \mathrm{WG}=27.7$ [4.09-173.1], 39-41 WG $=17.8$ [2.0-113.5]. Conclusion: $\mathrm{u}-\mathrm{NGAL}$ values in neonates seem to increase with gestational age and postnatal life hours; however, more studies are required to determine normal values.
\end{abstract}

\footnotetext{
*Correspondencia: KPP, karen.plascencia1@gmail.com

Conflicto de intereses: Los autores declaran que no tienen.

Citar como: Plascencia-Pimentel K, Bernárdez-Zapata I, Gerardo-Del Hoyo MN, Iglesias-Leboreiro J, Rendón-Macías ME, Romo-Vázquez $\mathrm{JC}$, et al. Niveles normales del marcador lipocalina asociada con gelatinasa de neutrófilo (NGAL) en orina en recién nacidos pretérmino y de término a las 24 y 72 horas de vida. Rev Mex Pediatr 2019; 86(6):223-228. doi: 10.35366/91873

[Normal levels of urine neutrophil gelatinase-associated lipocalin (u-NGAL) in pre-term and term newborns at 24 and 72 hours of life]
} 
neonatos parece aumentar conforme la edad gestacional y las horas de vida; sin embargo, se requieren más estudios para determinar los valores normales.

Palabras clave: Lipocalina asociada a gelatinasa de neutrófilo, niveles urinarios, neonatos, pretérmino, de término, biomarcador.

\section{INTRODUCCIÓN}

La insuficiencia renal aguda (IRA) es un incremento reversible de la creatinina en suero, asociada o no con reducción de la tasa de filtrado glomerular (TFG), ${ }^{1,2}$ aunada a una excreción inadecuada de productos de desecho con desequilibrio hidroelectrolítico y ácido-base. Su incidencia en neonatos en condiciones críticas varía entre 12.5 y $24 \%,{ }^{3}$ con una mortalidad de $10-61 \% .{ }^{1}$ Se han asociado múltiples factores, dentro de los prenatales se menciona la exposición materna a inhibidores de la enzima convertidora de angiotensina (IECAs), bloqueadores del receptor de angiotensina y antiinflamatorios no esteroideos (AINEs); mientras que de los postnatales se describen los prerrenales ( $85 \%$ de los casos) por disminución del volumen intravascular o del volumen sanguíneo, los renales (11\% de los casos) por necrosis tubular aguda, nefritis intersticial, necrosis por lesiones vasculares y por infecciones; en tanto que los postrenales ( $3 \%$ de los casos) se relacionan con anormalidades congénitas del riñón o de las vías urinarias. ${ }^{1}$

En neonatos, los datos clínicos de IRA aparecen tardíamente. ${ }^{4}$ El más temprano es la oliguria $(<1.0$
Keywords: Urinary neutrophil gelatinase-associated lipocalin; urinary levels, neonates, pre-term, term, biomarker.
$\mathrm{mL} / \mathrm{kg} / \mathrm{h}),{ }^{1}$ aunque es un dato que ayuda poco al diagnóstico. Otros pacientes iniciarán con hipertensión arterial. ${ }^{1,5}$ Un indicador de IRA es la elevación de la creatinina sérica; sin embargo, en las primeras 72 horas de vida ésta podría reflejar los niveles maternos. ${ }^{4}$ Además, los niveles se modifican por el sexo, la masa muscular, el metabolismo, el uso de drogas y otros componentes endógenos. ${ }^{6,7}$

El diagnóstico de IRA se ha basado en índices ${ }^{8}$ como la clasificación de la Acute Kidney Injury Network (AKIN por sus siglas en inglés); ${ }^{1}$ el sistema Risk, Injury, Failure, Loss End-Stage neonatal (nRIFLE por sus siglas en inglés); 4,8 y el cálculo de la tasa de filtración glomerular (TFG) a través de la fórmula de Schwartz ajustada a la edad gestacional. ${ }^{8}$ Para el cálculo de cualquiera de estos índices se requiere la medición de la creatinina sérica y cuantificar de manera adecuada la uresis. ${ }^{1,6-8,9}$

Recientemente se han propuesto algunos biomarcadores para IRA de fácil obtención, no invasivos, con alta sensibilidad y capaces de establecer los subtipos: prerrenal, intrarrenal y postrenal. ${ }^{1}$ Uno es la lipocalina asociada a gelatinasa de neutrófilo (NGAL) en orina. ${ }^{4,10}$

\begin{tabular}{|c|c|c|c|c|}
\hline & SEG & $n$ & Mediana (mín.-máx.) & IC $95 \%$ \\
\hline \multirow[t]{4}{*}{$\mathrm{NGAL}$ a las $24 \mathrm{~h}(\mathrm{ng} / \mathrm{mL})$} & $25-32$ & 25 & $18.2(0.5-543.1)$ & $11.4-110$ \\
\hline & $33-35$ & 25 & $22.3(0.9-84.4)$ & $8.7-25.5$ \\
\hline & $36-38$ & 10 & $10.1(2.5-181.9)$ & $5-99.2$ \\
\hline & $39-41$ & 10 & $20.4(0-577)$ & $11.5-560$ \\
\hline \multirow[t]{4}{*}{$\mathrm{NGAL}$ a las $72 \mathrm{~h}(\mathrm{ng} / \mathrm{mL})$} & $25-32$ & 25 & $47.2(7.5-441.2)$ & $26.1-95.4$ \\
\hline & $33-35$ & 25 & $33.0(4.7-235.5)$ & $17.3-84.4$ \\
\hline & $36-38$ & 10 & $27.7(4.09-173.1)$ & $9.7-82$ \\
\hline & $39-41$ & 10 & $17.8(2-113.5)$ & $3.6-87.6$ \\
\hline
\end{tabular}


Tabla 2: Niveles de NGAL urinarios en recién nacidos sin insuficiencia renal, descritos por diversos autores.

\begin{tabular}{|c|c|c|c|c|}
\hline Autor y año & Semanas edad gestacional & $\mathrm{n}$ & Días de vida & NGAL (ng/mL) \\
\hline & & & & $\min -\max$ \\
\hline \multirow[t]{6}{*}{ Roszkowska-Blaim M et al. 2013} & $\leq 26$ & ND & ND & $351(271-456)$ \\
\hline & $26.1-28$ & ND & ND & $158(117-212)$ \\
\hline & $28.1-30$ & ND & ND & $145(96-218)$ \\
\hline & 30.1 .36 & ND & ND & $85(53-134)$ \\
\hline & $>36.1$ & ND & 1 & $61.4(41.9-137.7)$ \\
\hline & & & 10 & $1.1(0.9-1.9)$ \\
\hline \multirow[t]{9}{*}{ Chen CN et al. 2015} & $29.2 \pm 2.4$ & 11 & 3 & $56.8(13-114)$ \\
\hline & & & 7 & $45.6(17-402)$ \\
\hline & & & 14 & $44.7(11-349)$ \\
\hline & & & 21 & $32.3(18-89)$ \\
\hline & $32.7 \pm 0.9$ & 13 & 3 & $37.6(8.6-108)$ \\
\hline & & & 7 & $34.1(5.4-259)$ \\
\hline & & & 14 & $39.8(6.3-94)$ \\
\hline & & & 21 & $28.2(4.7-310)$ \\
\hline & $39 \pm 1.1$ & 38 & 3 & $88.1(12-244)$ \\
\hline \multirow[t]{3}{*}{ Suchojad A et al. 2015} & $<29$ & 25 & 7 & $106(66-146)$ \\
\hline & & & 14 & $134(73-194)$ \\
\hline & & & 28 & $63(32-95)$ \\
\hline \multirow[t]{6}{*}{ Elmas AT et al. 2017} & $29.1 \pm 1.3$ & 51 & 1 & $1.47(0.15-10)$ \\
\hline & & & 3 & $2.6(0.15-10)$ \\
\hline & & & 7 & $2.93(0.15-10)$ \\
\hline & 30 & 32 & 1 & 106 (24-188) \\
\hline & & & 3 & $91(40-142)$ \\
\hline & & & 7 & $96(11-180)$ \\
\hline
\end{tabular}

NGAL es una proteína siderófora de 178 aminoácidos y $25-\mathrm{KDa},{ }^{3,6,11}$ que se expresa en bajas concentraciones en diferentes órganos. Su producción se incrementa en infecciones, procesos inflamatorios o cuando hay isquemia. ${ }^{12} \mathrm{El}$ daño isquémico de las células renales del asa de Henle y túbulo colector ${ }^{6,13}$ causa alta excreción urinaria de NGAL. ${ }^{2}$ En pacientes que desarrollan IRA, la relación NGAL sérica/urinaria elevada se ha asociado con incremento de la creatinina sérica $>1.2$ $\mathrm{mg} / \mathrm{dL}$ al día siguiente. ${ }^{11,14}$

Los niveles urinarios de NGAL $\leq 150 \mathrm{ng} / \mathrm{mL}$ (mínimo 100, máximo $270 \mathrm{ng} / \mathrm{mL}$ ) son considerados normales en niños y adultos. ${ }^{11,14,15}$ Los niveles en neonatos no se han establecido, particularmente en neonatos pretérmino, ya que en los estudios se han incluido pocos pacientes y con diferentes formas de establecer los límites de normalidad.

El objetivo de este estudio es presentar los valores de NGAL urinarios en neonatos pretérmino y de tér- mino sanos, a las 24 y 72 horas de vida, de acuerdo con la edad gestacional.

\section{MATERIAL Y MÉTODOS}

Se condujo un estudio prospectivo, y longitudinal con neonatos admitidos en la Unidad de Cuidados Intensivos Neonatales del Hospital Español de México, el cual es un hospital privado en la Ciudad de México. La captación de pacientes fue entre junio de 2017 y marzo de 2018.

Se incluyeron neonatos nacidos en este hospital, quienes fueron vigilados hasta su egreso hospitalario. Se excluyeron pacientes con malformaciones congénitas, datos de hipoxia neonatal moderada o severa, con menos de 25 semanas de edad gestacional (SEG) al nacimiento y si desarrollaron IRA dentro de las primeras 72 horas de vida (TFG $<11 \mathrm{~mL} / \mathrm{min} / 1.72 \mathrm{~m}^{2}$ en neonatos de 25 a 33 SEG y $<17 \mathrm{~mL} / \mathrm{min} / 1.72 \mathrm{~m}^{2}$, en $>34 \mathrm{SEG}$ ). ${ }^{16}$ 
La edad gestacional se determinó por fecha de última menstruación. La medición de TFG y N-GAL urinario se realizó a las 24 y 72 horas.

NGAL fue medida en orina ( $5 \mathrm{~mL})$, la cual recolectada por bolsa o sonda urinaria. Las muestras se procesaron en el laboratorio de nuestro hospital por técnica de quimioluminiscencia con reactivo de Abbot Architect Urine NGAL ${ }^{\circledR}$ (seis niveles de calibrador y tres niveles de control). Todas las muestras fueron procesadas antes de cumplirse 60 minutos de su recolección. Los valores son reportados en $\mathrm{ng} / \mathrm{mL}$.

El estudio fue aprobado por el comité de ética e investigación de nuestro hospital. Aunque se consideró un estudio sin riesgo por la toma de la orina, al no ser un procedimiento habitual se solicitó consentimiento informado a los padres.

\section{Análisis estadístico}

Para determinar los valores de referencia, los neonatos se agruparon según su edad gestacional al nacimiento: de 25 a 32, de 33 a 35, de 3 a 38 y de 39 a 41 SEG. Dado que no tuvieron distribución normal, en cada grupo se presentan los valores de NGAL en mediana, valores mínimo y máximo, así como en intervalo de confianza al 95\% (IC95\%). Los análisis se efectuaron con el paquete estadístico SPSS ${ }^{\circledR}$ versión 22.

\section{RESULTADOS}

De un total de 81 pacientes nacidos en este periodo de estudio se eliminaron 11 por desarrollar IRA antes de cumplir 72 horas de vida (Figura 1). También en esta figura se muestran las características de los recién nacidos.

\section{Valores de NGAL urinarios}

En la Tabla 1 se muestra la distribución de los valores encontrados en los 70 neonatos por grupos de edad gestacional a las 24 y 72 horas de vida. A las 24 horas

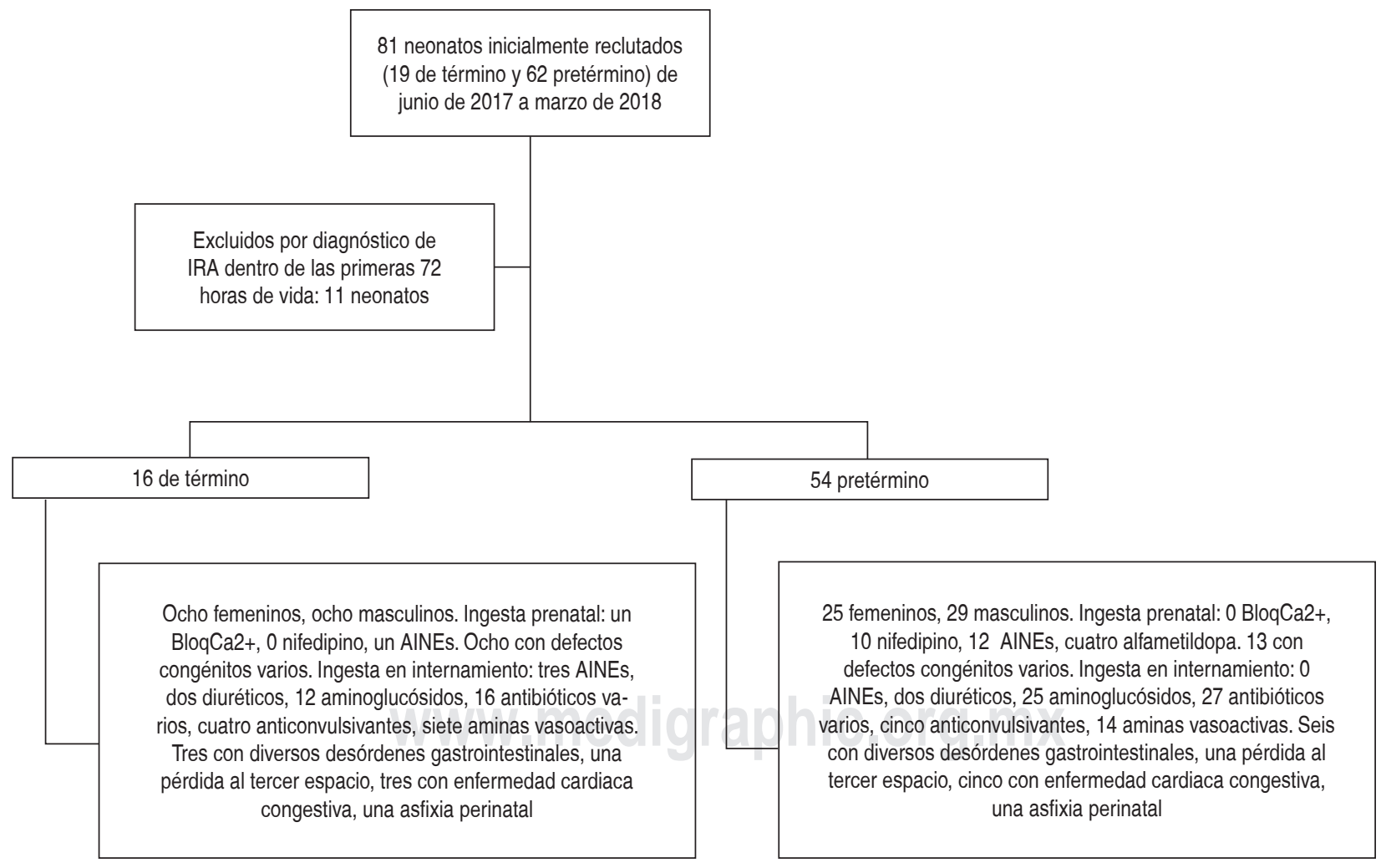

Figura 1: Características de los pacientes reclutados en el estudio según condición de pretérmino o de término al nacer. En el cuadro se anotan los antecedentes encontrados en cada grupo.

IRA = insuficiencia renal aguda; BloqCa2+ = bloqueadores de calcio; AINEs = antiinflamatorios no esteroideos. 
los valores no parecen diferentes de acuerdo con la edad gestacional, mientras que a las 72 horas los valores tienden a aumentar, con excepción de los neonatos de 39-41 SEG.

\section{DISCUSIÓN}

En este estudio presentamos valores normales de NGAL urinarios en recién nacidos sanos tanto de término como de pretérmino en sus primeras 24 y 72 horas de vida. En todos se cuidó que los participantes no tuvieran evidencia de enfermedad renal. Para la presentación de los datos, se calculó la mediana de los valores y sus valores extremos con el objetivo de comparar los valores con otros estudios. $1,3,11,14$

De los estudios encontrados, nuestras cifras en general no son similares, ubicándose tanto por arriba (cerca de 10 veces más altos) ${ }^{3}$ como por debajo (cerca de ocho veces más bajos). ${ }^{1,11,14}$ En el estudio de Roszkowska y colaboradores, ${ }^{1}$ quienes midieron los valores a las 24 horas y por grupos de semanas de gestación, encontraron valores por arriba de 80 $\mathrm{ng} / \mathrm{mL}$ en neonatos pretérmino < $36 \mathrm{SEG}$, además de observar una disminución de las cifras conforme aumentó la edad gestacional. En nuestros pacientes menores de 36 SEG no se apreció una reducción en los neonatos con más semanas de gestación. Para los neonatos de término, también en el estudio de Roszkowska ${ }^{1}$ se informaron cifras alrededor de $61 \mathrm{ng} /$ $\mathrm{mL}$, valor menor que el que nosotros encontramos. También observamos discrepancia con los valores informados por Elmas, ${ }^{3}$ los cuales fueron 10 veces más bajos que nuestros prematuros < 31 SEG a las 24 horas de vida.

Para los valores a las 72 horas de vida, sólo para los neonatos de 31 a 34 SEG evaluados por Chen y su equipo ${ }^{14}$ tuvieron cifras semejantes a las nuestras (Tabla 2), los otros grupos de edad gestacional fueron diferentes a los hallazgos de este estudio. En el estudio de Suchojad y colegas ${ }^{11}$ los valores tanto en prematuros como en recién nacidos de más de 30 SEG a las 72 horas se observaron cifras promedio de $106 \mathrm{ng} / \mathrm{mL}$.

La cantidad de pacientes que estudiamos para cada edad gestacional fue mayor que la reportada en estudios previos. ${ }^{1,3,11,14}$ Sin embargo, en algunos otros estudios ha sido mayor el periodo de recolección de valores de NGAL urinario, prolongándose de 10 días ${ }^{1,3}$ y hasta 21 y 28 días. ${ }^{11,14}$ Un aspecto interesante es que en estudios donde se han evaluado a más largo plazo, los niveles de NGAL urinario permanecen estables después de las 72 horas de vida. De ahí que para este estudio consideramos sólo realizar el seguimiento hasta este tiempo.

Un aspecto a señalar es que las cifras que se informan en este estudio, están por debajo de los niveles descritos en estudios de neonatos con falla renal, cuyos valores suelen ser superiores a $500 \mathrm{ng} / \mathrm{mL} .1,3,11,14$ Como se comentó en los antecedentes, la lesión en las células del asa de Henle suele causar excreciones importantes de este biomarcador. ${ }^{6,13}$

A pesar del mayor número de pacientes en comparación con estudios previos, es necesario aceptar que en este estudio hubo pocos participantes en los diferentes grupos de edad gestacional, condición que puede explicar la amplia variabilidad detectada (Tabla 1).

Finalmente, debido a los diferentes datos que se han reportado hasta ahora de NGAL urinario en neonatos, parece necesario llevar a cabo mayor número de estudios para determinar con precisión los valores de referencia en neonatos.

\section{AGRADECIMIENTOS}

Al Quím. José Luis Rodríguez Vázquez, jefe del Laboratorio Clínico del Hospital Español por su apoyo total en la realización de este estudio.

\section{REFERENCIAS}

1. Roszkowska-Blaim M, Kisiel A. Role of biomarkers in the early diagnosis of acute kidney injury in neonates. Postpy Nauk Med. 2013; 26(2): 138-143.

2. Libório AB, Branco KM, Torres de Melo-Bezerra C. Acute kidney injury in neonates: from urine output to new biomarkers. Biomed Res Int. 2014; 2014: 601568.

3. Elmas AT, Karadag A, Tabel Y, Ozdemir R, Otlu G. Analysis of urine biomarkers for early determination of acute kidney injury in non-septic and non-asphyxiated critically ill preterm neonates. $J$ Matern Fetal Neonatal Med. 2017; 30(3): 302-308.

4. Smertka M, Wroblewska J, Suchojad A, Majcherczyk M, JadamusNiebroj D, Owsianka-Podlesny T, et al. Serum and urinary NGAL in septic newborns. Biomed Res Int. 2014; 2014: 717318.

5. Parravicini E, Locatelli C, Lorenz JM, Nemerofsky SL, Bateman DA. Is urinary neutrophil gelatinase-associated lipocalin able to predict acute kidney injury episodes in very low birth weight infants in clinical settings? Pediatr Res. 2016; 80(5): 663-667.

6. El-Salam MA, Zaher MM, Mohamed RAE, Shall LYA, Saleh RAM, Hegazy AA. Comparison of some urinary biomarkers of acute kidney injury in term new born with and without asphyxia. Clin Med Diagnostics. 2014; 4(2): 23-28.

7. Izadi A, Yousefifard M, Nakhjavan-Shahraki B, Baikpour M, Razaz JM, Hosseini M. Diagnostic value of urinary neutrophil gelatinaseassociated lipocalin (NGAL) in detection of pediatric acute kidney injury; a systematic review and meta-analysis. Int J Pediatr. 2016; 4(11): 3875-3895.

8. García-Pérez CS, Cordero-González G. Función renal en el recién nacido. Perinatol Reprod Hum. 2011; 25(3): 161-168. 
9. Askenazi DJ, Koralkar R, Hundley HE, Montesanti A, Parwar P, Sonjara $S$ et al. Urine biomarkers predict acute kidney injury in newborns. J Pediatr. 2012; 161: 270-250.

10. El Frargy MS, Soliman NA. Urinary neutrophil gelatinase associated lipocalin and interleukin-18 as early predictors of kidney injury in neonates. J Mol Biomark Diagn. 2016; 3.

11. Suchojad A, Tarko A, Smertka M, Majcherczyk M, Brzozowska $A$, Wroblewska $J$ et al. Factors limiting usefulness of serum and urinary NGAL as a marker of acute kidney injury in preterm newborns. Ren Fail. 2015; 37(3): 439-445.

12. Sellmer A, Bech BH, Bjerre JV, Schmidt MR, Hjortdal VE, Esberg $G$ et al. Urinary Neutrophil Gelatinase-associated Lipocalin in the evaluation of Patent Ductus Arteriosus and AKI in Very Preterm Neonates: a cohort study. BMC Pediatr. 2017; 17(1): 7.
13. Kuribayashi R, Suzumura $H$, Sairenchi $T$, Watabe $Y$, Tsuboi $Y$, Imataka $G$ et al. Urinary neutrophil gelatinase-associated lipocalin is an early predictor of acute kidney injury in premature infants. Exp Ther Med. 2016; 12(6): 3706-3710.

14. Chen $\mathrm{CN}$, Chou CH, Jeng SF, Tsai IJ, Chen PC, Chen CY et al. Urinary neutrophil gelatinase-associated lipocalin levels in neonates. Pediatr Neonatol. 2016; 57(3): 207-212.

15. Albeladi FI, Algethamy HM. Urinary Neutrophil GelatinaseAssociated lipocalin as a predictor of acute kidney injury, severe kidney injury, and the need for renal replacement therapy in the Intensive Care Unit. Nephron Extra. 2017; 7(2): 62-77.

16. Tschudy MM, Arcara KM. Manual Harriet Lane de pediatría. 19a ed. España: Ed. Elsevier; 2013. 